\title{
Verzeichnis und Erklärung der benutzten Fremdwörter und kriminologischen Fachausdrücke \\ (soweit sie im Text nicht erläutert sind)
}

\author{
Aberration \\ Ätiologie, ätiologisch \\ Affekt, affektiv \\ Akzeleration \\ akzidentell \\ Animismus, \\ animistisch \\ Anomie, anomisch \\ Anthropologie, anthro- \\ pologisch \\ Antinomie \\ Anthropometrie, anthro- \\ pometrisch \\ Anus, anal \\ Apriorismus, apriorisch \\ Arteriosklerose, arterio- \\ sklerotisch \\ Asthenie, asthenisch \\ Atavismus, atavistisch \\ Atrophie, atrophisch \\ Bagatellkriminalität
}

Behaviorismus, behavioristisch

Chiffren

Chromosom

Daktyloskopie

Darwinismus

Demographie, demographisch

Demoskopie, demoskopisch

\author{
Abweichung von der Normalform \\ Lehre von den Ursachen \\ heftige Erregung, Zustand einer außergewöhnlichen \\ seelischen Angespanntheit \\ Beschleunigung des Entwicklungsprozesses, Vorverlegung \\ der körperlichen und (oder) psychischen Wachstums- und \\ Entwicklungsreife (Gegensatz: Retardierung) \\ zufällig, gelegentlich \\ Glaube an seelische Mächte, an Geister
}

Normlosigkeit, Zustand mangelnder sozialer und psychischer Ordnung (Gegensatz: Synnomie)

Wissenschaft vom Menschen und seiner Entwicklung in natur- und geisteswissenschaftlicher Hinsicht

Widerspruch zweier Lehrmeinungen, von denen jede

Gültigkeit beansprucht

Lehre von den Maßverhältnissen am menschlichen Körper

After

Lehre von der Erkenntnis, die unabhängig von der Erfahrung vorgegeben ist

krankhafte Veränderung der Arterien, Arterienverkalkung

Kraftlosigkeit, Schwäche, Kräfteverfall

Entwicklungsrückschlag

Organschwund

Verstöße gegen Strafrechtsnormen, die geringen materiellen, körperlichen, psychischen und sozialen Schaden anrichten und die von der Bevölkerung als unbedeutend angesehen werden

Richtung der Lernpsychologie, die dem

Reiz-Reaktions-Modell zentrale Bedeutung beimißt verschlüsselte Zeichen, Geheimzeichen

Träger von Erbanlagen

Fingerabdruckverfahren

von dem englischen Naturforscher Charles Darwin

(1809-1882) begründete Lehre von der

stammesgeschichtlichen Entwicklung durch Auslese

Bevölkerungswissenschaft

Meinungsumfrage, -forschung 
XXVI Verzeichnis der benutzten Fremdwörter und kriminologischen Fachausdrücke

Deprivation

Desperado

Diversion

dynamisch

dysfunktional

Egozentrismus, egozen-

trisch

Einstellung

Eklektizismus, eklektisch

Elektroenzephalogramm

Embryologie

Emotion, emotional endokrin

Endokrinologie, endo-

krinologisch

ethnisch

Exploration, explorieren

extra-, intrauterin

Feminismus, feministisch

Fetus (Fötus)

Frustration

Frustrationstoleranz

Funktion, funktional

genetisch

Genitalien, genital

Geriatrie, geriatrisch

Gerontologie, gerontologisch
Mangel, Verlust, Entzug von etwas Erwünschtem, fehlende Zuwendung, Liebesentzug

ein zu jeder Verzweiflungstat Entschlossener

Umleitung des Straftäters um das Kriminaljustizsystem herum, informelle außergerichtliche Regelung krimineller oder delinquenter Konflikte

durch inneren Antrieb sich bewegend

(Gegensatz statisch $=$ feststehend)

den funktionalen Ablauf störend

Ichbezogenheit, Weltauffassung, die alles in bezug auf die eigene Person wertet

Motivationsbereitschaft

unoriginelle geistige Arbeitsweise, bei der Ideen anderer

zusammengetragen und übernommen werden

Aufzeichnung des Verlaufs der Hirnaktionsströme

Wissenschaft von der vorgeburtlichen Entwicklung der

Lebewesen

Gefühlszustand, Gemütsbewegung

die nach innen gerichtete Absonderung betreffend

Lehre von den endokrinen Drüsen

einer sprachlich und kulturell einheitlichen Volksgruppe

angehörend

Gespräch mit dem Probanden

außerhalb und innerhalb der Gebärmutter

Frauenbewegung, die eine grundlegende Veränderung der

traditionellen Rollenverteilung zwischen Mann und Frau und

die Beseitigung des Patriarchats, der bevorzugten Stellung des Mannes in Staat, Wirtschaft und Gesellschaft, anstrebt menschliche Leibesfrucht vom dritten

Schwangerschaftsmonat an

Erlebnis der wirklichen oder vermeintlichen Benachteiligung bei enttäuschter Erwartung oder bei erlittener

Ungerechtigkeit

Fähigkeit, eine Versagung über längere Zeit auszuhalten, ohne sie durch Verdrängung ins Unbewußte

fehlzuverarbeiten oder unmittelbar aggressiv zu reagieren

die Leistung, der Beitrag oder die erkennbare Konsequenz

eines sozialen Elements für den Aufbau, die Erreichung,

Erhaltung oder Veränderung eines bestimmten Zustandes des gesamten Systems, zu dem das Element gehört

die Entstehung, Entwicklung der Lebewesen betreffend, entwicklungsgeschichtlich

die männlichen und weiblichen Geschlechtsorgane

Teilgebiet der Psychiatrie, das sich mit Alterskrankheiten befaßt

Wissenschaft vom menschlichen Altern, die sich mit allen Phänomenen auseinandersetzt, die Begleiterscheinungen 
Hedonismus

Heterogenität, heterogen

Heterostereotyp

Heuristik, heuristisch

Identifikation

Innovation
in re
Instinkt, instinktiv
Interaktion
Interdependenz
Interiorisation
irrational
Internalisation, internali-
sieren

interpsychisch intrapsychisch

Introjektion

irreversibel

Kapitalverbrechen

Kavaliersdelikt

Kognition, kognitiv

Kohabitation, kohabitieren

Kohorte

Kommunikation

Konflikt oder Folgen des höheren Lebensalters sind

philosophische Lehre, die die sinnliche Lust, das Vergnügen,

den Genuß als Motiv, Ziel oder Beweis alles sittlichen

Handelns betrachtet

Verschiedenartigkeit, Uneinheitlichkeit

Vorurteil, das Mitglieder einer Gruppe von einer anderen

Gruppe besitzen

Wissenschaft von den Verfahren, Probleme zu lösen, methodische Anleitung zur Gewinnung neuer Erkenntnisse die unbewußte Angleichung an das Wesen und Verhalten eines anderen Menschen, meist aufgrund der (unbewußten)

Nachahmung von Vorbildern

Neuerung

in der Sache, im Rechtsstreit, im Verfahren

angeborene, keiner Übung bedürfende Verhaltensweise und Reaktionsbereitschaft

Wechselbeziehung, gegenseitig aufeinander bezogene

Handlungen, aktive Reaktion

gegenseitige Abhängigkeit

Verinnerlichung

nicht verstandesgemäß, vernunftwidrig

Hereinnahme fremder Einstellungen, Überzeugungen,

Motive und soziokultureller Muster (Werte, Normen, Erwartungen) in das kognitive (Denk-) und das affektive (Gefühls-)System der Persönlichkeit

zwischenmenschlich

innerseelisch

Einverleibung fremder Anschauungen und Motive in die

Persönlichkeit (Gegensatz: Projektion)

nicht umkehrbar, nicht rückgängig zu machen

Straftaten, die schwere körperliche, wirtschaftliche, seelische und soziale Schäden anrichten und die von der Bevölkerung als Schwerkriminalität beurteilt werden, z. B. Mord, Raub, Vergewaltigung

Straftat, die zwar schweren wirtschaftlichen, gesundheitlichen und sozialen Schaden anrichten kann, die von der Bevölkerung aber als akzeptabel, als nichtkriminell angesehen wird

psychische Vorgänge, die mit dem Erkennen zusammenhängen, z. B. Wahrnehmung, Erinnerung, Vermutung, Erwartung, Problemlösen

Geschlechtsverkehr

Gruppe von Menschen, die im selben Zeitabschnitt geboren sind und die man auswählt, um die Einflüsse der Faktoren zu ermitteln, die im Laufe der Zeit auf ihr Verhalten einwirken Prozeß der Informationsübertragung Zusammenstoß, Widerstreit zwischen Personen, Gruppen, Gesellschaften, Staaten über Werte, Ziele, Machtverhältnisse 
XXVIII Verzeichnis der benutzten Fremdwörter und kriminologischen Fachausdrücke

Konquistador

Konstitution

Korrelation, korrelieren

Kortex, kortikal

Labilität, labil

latent

Legalbewährung

Magna Charta

Masturbation

Moratorium

Mortalität

Motiv

Motivation

Nahraum (sozialer)

Narzißmus, narzißtisch

Neurasthenie

Neurophysiologie, neurophysiologisch

obskur

Okkultismus

Ontogenese, ontogenetisch

oral

Orgasmus

Orthodoxie, orthodox

Ottoman, ottomanisch

Phänomenologie, phänomenologisch

Phrenologie
Teilnehmer an der spanischen Eroberung Südamerikas im 16. Jahrhundert

körperliche und seelische Verfassung des Menschen

Ausmaß, in dem zwei oder mehr veränderliche Merkmale voneinander abhängig streuen

Großhirnrinde

Beeinflußbarkeit, Schwäche (Gegensatz: Stabilität)

vorhanden, ohne äußerlich erkennbar zu sein (Gegensatz zu manifest: äußerlich sichtbar)

keine Rückfälligkeit nach Strafverbüßung im Sinne eines

Verstoßes gegen Strafgesetze

Grundgesetz von 1215, in dem der englische König dem Adel grundlegende Freiheitsrechte garantieren mußte

geschlechtliche Selbstbefriedigung

gesetzlich angeordneter oder vertraglich vereinbarter

Aufschub

Verhältnis der Todesfälle an einer Erkrankung, bezogen auf die Zahl der Gesamtbevölkerung

der bewegende, richtunggebende, leitende, antreibende

seelische Hinter- und Bestimmungsgrund des Handelns

(Triebfeder des Wollens)

Struktur aktivierender und richtunggebender

Bestimmungsgründe, die für die Auswahl und Stärke der

Aktualisierung von Verhaltensbereitschaften bedeutsam sind

Bereich, in dem der persönliche Kontakt und die Interaktion von Person zu Person möglich ist, z. B. in der Familie, Schule, in der Berufs- und Freizeitgruppe. Der soziale Fernraum, in dem Angesicht-zu-Angesicht-Kontakte wegfallen, übt gleichwohl auch bedeutsame Einflüsse auf die Person, z. B. durch die Massenmedien, aus

Verliebtsein in sich selbst, Selbstliebe nervöse Erschöpfung, Nervenschwäche

Wissenschaft von den Lebensvorgängen und Funktionen des Nervensystems

dunkel, verdächtig, zweifelhafter Herkunft

Lehren und Praktiken, die sich mit der Wahrnehmung

übersinnlicher Kräfte beschäftigen

Entwicklung des Individuums von der Eizelle zum

geschlechtsreifen Zustand

den Mund betreffend

Höhepunkt der geschlechtlichen Erregung

Rechtgläubigkeit; im negativen Sinne auch: engstirniges

Festhalten an einer Lehrmeinung

Bezeichnung nach Osman, dem Begründer des türkischen

Herrscherhauses der Ottomanen

Lehre von den Erscheinungen, den Phänomenen

wissenschaftlich widerlegte Lehre, die aus der Schädelform 
Phylogenese, phylogenetisch

Physiognomie, physiognomisch

Polemik, polemisch

Polygraph

Polymorphie, polymorph

Population

Prädestination

Prädisposition

proaktiv

Proband

Prohibition

Projektion

Promiskuität

prospektiv

Puritanismus, Puritaner, puritanisch

Quäker

Rassismus

reaktiv

Repression, repressiv

Retardierung

Ressource

retrospektiv

Ritual, rituell

Sanktion, Sanktionie-

rung

Schicht auf bestimmte geistig-seelische Anlagen schließen wollte

Stammesgeschichte der Lebewesen

Gesichtsausdruck eines Menschen

unsachlicher Angriff, ins Persönliche gehende Kritik "Lügendetektor", Gerät zur gleichzeitigen Registrierung mehrerer Vorgänge und Erscheinungen

Vielgestaltigkeit

Gesamtheit der Träger eines Merkmals oder aller Meßwerte

Vorherbestimmung

Anlage, Empfänglichkeit

von sich selbst aus tätig werdend, selbständig, unabhängig handelnd

Versuchsperson, die sich in ihrer Leistungsfähigkeit und

Persönlichkeitsstruktur zu „erweisen“ (lat. probare) hat

staatliches Verbot der Herstellung und des Verkaufs

alkoholischer Getränke

Übertragung und Zuschreibung eigener unerwünschter

Motive und Eigenschaften auf andere

Geschlechtsverkehr mit verschiedenen, häufig wechselnden

Partnern

vorausschauend

englische protestantische Bewegung, die der Kirche in Liturgie, Lehre und Verfassung eine „reine“ Form geben wollte. Evangelische Reinheit der Lebensführung, strikte Sonntagsheiligung und religiöses Berufsethos zeichneten ihre Anhänger aus. Die Puritaner prägten durch ihre

Einwanderung die Geistesart und den Lebensstil der

Bevölkerung Nordamerikas.

ursprünglich Spottname, evangelische Sekte, "Gesellschaft

der Freunde", die dank ihrer brüderlichen und sozialen

Gesinnung für die Sklavenbefreiung, die Gleichberechtigung

der Frau, den Weltfrieden eintraten und die nach beiden

Weltkriegen durch große Hilfsleistungen hervorgetreten sind

Einstellung, die einen Menschen allein wegen seiner Rasse

benachteiligt und diskriminiert

auf Handlungen anderer tätig werdend, unselbständig, von

anderen abhängig handelnd

Unterdrückung

Entwicklungsverzögerung (Gegensatz: Akzeleration)

Hilfsmittel, Hilfsquelle

rückschauend, rückblickend

Vorgehen nach festgelegter, förmlicher Ordnung

gesellschaftliche, mißbilligende Reaktion auf

sozialabweichendes Verhalten

Bevölkerungsgruppe, deren Mitglieder bestimmte

gemeinsame Merkmale besitzen und sich dadurch von 
senil

Sensibilität, sensibilisie-

ren

Sequenz

Sexismus

Signifikanz, signifikant

Simulation

somatisch

Stereotyp

Stigmatisierung, Stigmatisation Sozialstruktur, sozialstrukturell

Substrat

Symbol, symbolisch

Synnomie

Synthetikum

System

Telepathie anderen Bevölkerungsgruppen in einer Sozialstruktur unterscheiden, die durch eine feste Rangordnung gekennzeichnet ist. Die soziale Schicht kann man objektiv durch Familieneinkommen und Beruf des Familienvaters oder subjektiv durch eigene Zurechnung zu einer Schicht und Zustimmung zu deren Wertvorstellungen und Leitbildern bestimmen

greisenhaft, altersschwach

Empfindsamkeit, Feinfühligkeit

Aufeinanderfolge, Abfolge

Einstellung, die einen Menschen allein wegen seines

Geschlechts benachteiligt und diskriminiert

Bezeichnung für die Sicherheit oder Wahrscheinlichkeit, mit der angenommen werden kann, daß bestimmte Unterschiede zwischen Stichproben oder Teilgesamtheiten einer Stichprobe sowie bestimmte Größen wie etwa

Korrelationskoeffizienten nicht zufällig, durch die Zufallsauswahl bedingt, sondern Kennzeichen der untersuchten Grundgesamtheiten sind. Als signifikant werden aufgrund eines Signifikanztests solche Ergebnisse bezeichnet, die mit sehr hoher Wahrscheinlichkeit nicht auf dem Auswahlfehler der Zufallsauswahl beruhen

Nachahmung

auf den Körper bezogen, körperlich

festgefügte, für lange Zeit gleichbleibende, durch neue

Erfahrungen kaum veränderbare, meist positiv oder negativ bewertende und emotional gefärbte Vorstellung über Personen und Gruppen, Ereignisse und Gegenstände Brandmarkung

Gliederung eines sozialen Systems nach bestimmten

Merkmalen, z. B. nach Einkommen, Bildung,

Produktionsverhältnissen, sozialer Schichtung, nach Prestige und Macht

Unterlage, Grundlage

Zeichen, Kennzeichen, Sinnbild, das im Rahmen einer Interaktion eine Bedeutung ausdrückt

Zusammenhalt, Übereinstimmung, Teilhabe an gemeinsamen Werten, Normen und Bräuchen (Gegensatz: Anomie) zusammengesetztes Kunstprodukt

nach einem einheitlichen Prinzip geordnetes Ganzes (statisch), ein Ganzes, dessen Elemente miteinander in wechselseitigen Beziehungen stehen, und zwar derart, daß jede Veränderung eines Elements auf andere Elemente im System fortwirkt (dynamisch)

Wahrnehmen seelischer Vorgänge eines anderen Menschen ohne Vermittlung der Sinnesorgane 
Theokratie, theokratisch

Totem

Totemismus, totemistisch

Trauma, traumatisch, traumatisieren

Triebe

Ubiquität, ubiquitär

Vagant, Vagabund

Vä́na, vaginal

Verbalisierung, verbal, verbalisieren

Voyeur

Wergeld

Zetetiker
"Gottesherrschaft", bei der der Herrscher die Staatsgewalt als Statthalter für Gott ausübt

Wesen oder Ding, das als Ahne gilt, als zauberischer Helfer verehrt wird und nicht verletzt werden darf

Glaube an die übernatürliche Kraft eines Totems

Wunde, Verletzung, insbesondere seelische Schädigung

erlebte Instinkte (Karl Jaspers), leibnahe Vorgänge, die auf ein Ziel hindrängen, dessen Erreichung mit Spannungslösung und Lustgewinn verbunden ist (Triebbefriedigung)

Allgegenwart, Gleichverteilung

umherziehender Mensch, Herumtreiber

weibliche Scheide

das Ausdrücken von Gedanken, Gefühlen, Wertvorstellungen in Worten

Zuschauer, Mensch mit der perversen Neigung, sexuelle Vorgänge bei anderen zu beobachten

Sühnegeld für eine kriminelle Tötung im germanischen Recht Zweifler, Wissenschaftler, der nicht an Dogmen glaubt oder in statischen Systemen, sondern in Problemen denkt 